Bull. Korean Math. Soc. 51 (2014), No. 5, pp. 1453-1467

http://dx.doi.org/10.4134/BKMS.2014.51.5.1453

\title{
SOME RESULTS RELATED TO COMPLEX DIFFERENTIAL-DIFFERENCE EQUATIONS OF CERTAIN TYPES
}

\author{
Kai LiU and Xianjing Dong
}

\begin{abstract}
In this paper, we consider the growth and existence of solutions of differential-difference equations of certain types. We also consider the differential-difference analogues of Brück conjecture and give a short proof on a theorem given by Li, Yang and Yi [18]. Our additional purpose is to explore the similarity or difference on some problems in differential, difference and differential-difference fields.
\end{abstract}

\section{Introduction}

We assume that the reader is familiar with standard symbols and fundamental results of Nevanlinna theory [12,21], which is an efficient tool in considering the growth and existence of solutions of differential equations [15]. Recently, difference analogues of Nevanlinna theory were established, such as $[7,9,10]$, which can be used to consider the growth and existence of solutions of difference equations, such as $[4,5,7,10,11]$.

In this paper, $f(z+c)$ is the shift of $f(z)$ and $f(z+c)-f(z)$ is the difference of $f(z)$. If an equation includes derivatives, shifts or differences of $f(z)$, then the equation is called differential-difference equation, which can be called DDE for short. Hence, it is interesting to consider the applications of Nevanlinna theory in the growth and existence of solutions of DDE. Combining the lemma of logarithmic derivatives $[12,21]$ with difference analogue of the lemma of logarithmic derivatives [11, Theorem 5.6], the key result for meromorphic functions with finite order is easily obtained,

$$
m\left(r, \frac{f^{\prime}(z)}{f(z+c)}\right) \leq m\left(r, \frac{f^{\prime}(z)}{f(z)}\right)+m\left(r, \frac{f(z)}{f(z+c)}\right)=S(r, f),
$$

Received June 26, 2013; Revised March 7, 2014.

2010 Mathematics Subject Classification. Primary 39B32, 34M05, 30D35.

Key words and phrases. entire solutions, differential-difference equations, finite order.

This work was partially supported by the NSFC (No. 11301260, 11101201), the NSF of Jiangxi (No. 20132BAB211003, 20122BAB211002) and the YFED of Jiangxi (No. GJJ13078) of China. 
where $S(r, f)$ is used to denote any quantity satisfies $S(r, f)=o(T(r, f))$, and $r \rightarrow \infty$ outside of a possible exceptional set of finite logarithmic measure. The equation (1) takes an important part in considering the value distribution of solutions of DDE.

This paper is organized as follows. In Section 2, we will consider which types of DDE can admit transcendental entire solutions. In Section 3, we will consider different types DDE of certain forms. In Section 4, we will consider the differential-difference analogues of Brück conjecture.

\section{Existence of solutions of differential-difference equations}

The Malmquist-Yosida theorem [15, Theorem 10.2] states that if the differential equation

$$
f^{\prime}(z)=R(z, f(z))
$$

admits a transcendental meromorphic solution $f(z)$, then it should be reduced into a Riccati differential equation $f^{\prime}(z)=a(z)+b(z) f(z)+c(z) f(z)^{2}$, if $(2)$ admits a transcendental entire solution $f(z)$, it should be reduced into linear differential equation $f^{\prime}(z)=a(z)+b(z) f(z)$, where $a(z), b(z), c(z)$ are small functions with respect to $f(z)$.

Similar results in difference, we recall the following theorem, which also be reconsidered in [7, Theorem 9.1] with a different proof.

Theorem A ([13, Proposition 8]). Let $c_{1}, \ldots, c_{n} \in \mathbb{C} \backslash\{0\}$. If the difference equation

$$
\sum_{i=1}^{n} f\left(z+c_{i}\right)=R(z, f(z))=\frac{a_{0}(z)+a_{1}(z) f(z)+\cdots+a_{p}(z) f(z)^{p}}{b_{0}(z)+b_{1}(z) f(z)+\cdots+b_{q}(z) f(z)^{q}}
$$

with small coefficients $a_{i}(z), b_{j}(z)$ with respect to $f$, admits a transcendental meromorphic solution with finite order, then $\max \{p, q\} \leq n$.

We should see that Theorem A is not valid for meromorphic functions with infinite order, which can be seen by $f(z)=e^{e^{z}}$ satisfying $f(z+\ln p)=f(z)^{p}$, where $p \geq 2$ is an integer. The fundamental reason is the difference analogue of logarithmic derivative lemma is not valid for meromorphic functions of infinite order, thus

$$
T(r, f(z+c))=T(r, f)+S(r, f)
$$

may not true. Using the difference analogues of logarithmic derivative lemma $[7,9]$, the equation (3) is true for meromorphic functions with finite order. It can be stated as follows.

Lemma 2.1. Let $f(z)$ be a transcendental meromorphic function of finite order. Then

$$
T(r, f(z+c))=T(r, f)+S(r, f)
$$


The Valiron-Mohon'ko theorem plays an important part in proving Theorem $\mathrm{A}$ and in the following, which can be stated as follows.

Lemma 2.2 ([15, Theorem 2.2.5]). Let $f$ be a meromorphic function. For all irreducible rational functions in $f$,

$$
R(z, f)=\frac{P(z, f)}{Q(z, f)}=\frac{\sum_{i=0}^{p} a_{i}(z) f^{i}}{\sum_{j=0}^{q} b_{j}(z) f^{j}}
$$

with meromorphic coefficients $a_{i}(z), b_{j}(z)$ such that $T\left(r, a_{i}(z)\right)=S(r, f)$ and $T\left(r, b_{j}(z)\right)=S(r, f)$, then $T(r, R(z, f))=d T(r, f)+S(r, f)$, where $d=\max \{p, q\}$.

But we cannot obtain $T(r, R(z, f(z+c)))=d T(r, f)+S(r, f)$ for meromorphic functions with infinite order, which can be seen by the function $f(z)=$ $e^{e^{z}}+\frac{1}{e^{e^{z}}}$, where $e^{c}=\frac{1}{2}$, here $T\left(r, f(z+c)^{2}\right)=T(r, f)+S(r, f)$. In fact, for the function $f(z)=e^{e^{z}}$, we have $T(r, f(z+c))=e^{c} T(r, f)+S(r, f)$, where $e^{c}$ can be an arbitrarily constant. However, for finite order meromorphic functions, we can obtain a similar result as Theorem A in DDE. It seems that we can consider the following two different types DDE

$$
\sum_{k=1}^{n} f^{(k)}(z)=R(z, f(z+c))=\frac{a_{0}(z)+a_{1}(z) f(z+c)+\cdots+a_{p}(z) f(z+c)^{p}}{b_{0}(z)+b_{1}(z) f(z+c)+\cdots+b_{q}(z) f(z+c)^{q}}
$$

and

(6) $\sum_{i=1}^{n} f\left(z+c_{i}\right)=R\left(z, f^{(k)}(z)\right)=\frac{a_{0}(z)+a_{1}(z) f^{(k)}(z)+\cdots+a_{p}(z) f^{(k)}(z)^{p}}{b_{0}(z)+b_{1}(z) f^{(k)}(z)+\cdots+b_{q}(z) f^{(k)}(z)^{q}}$,

where the right-hand side of above are rational in both $\operatorname{arguments}, a_{i}(z), b_{j}(z)$, $(i=1, \ldots, p, j=1, \ldots, q)$ are small functions with respect to $f$ and $c, c_{i}$ are non-zero constants. Remark that above two equations just have a little difference with (2), it is natural to explore the difference or similarity among (2), (5) and (6).

Theorem 2.3. Let $k$ be a positive integer. If (5) admits a transcendental meromorphic solution of finite order, then $\max \{p, q\} \leq k+1$. If (5) admits a transcendental entire solution of finite order, then $\max \{p, q\} \leq 1$.

Proof. Assume that $f$ is a transcendental meromorphic solution of finite order, using Lemma 2.2, then we have

$$
\begin{aligned}
\max \{p, q\} T(r, f(z+c)) & =T\left(r, \sum_{k=1}^{n} f^{(k)}(z)\right)+S(r, f) \\
& \leq m\left(r, \sum_{k=1}^{n} f^{(k)}(z)\right)+N\left(r, \sum_{k=1}^{n} f^{(k)}(z)\right)+S(r, f) \\
& \leq m(r, f)+(k+1) \bar{N}(r, f)+S(r, f) \\
& \leq(k+1) T(r, f)+S(r, f) .
\end{aligned}
$$


Combining above with Lemma 2.1, we have $\max \{p, q\} \leq k+1$. Obviously, if $f(z)$ is entire, then $\max \{p, q\} \leq 1$.

Example 1. The function $f(z)=e^{z}$ and $c_{1}=i \pi$ and $c_{2}=2 i \pi$, thus $f(z+$ $\left.c_{1}\right)+f\left(z+c_{2}\right) \equiv 0$, but $R\left(z, f^{(k)}(z)\right) \not \equiv 0$, otherwise $T\left(r, f^{(k)}\right)=S\left(r, f^{(k)}\right)$, which is impossible.

Remark 1. Example 1 shows that there is no a similar result as Theorem A or Theorem 2.3 for DDE (6). From the following two examples, we see that Theorem 2.3 is not valid for meromorphic functions with infinite order.

Example 2. The function $f(z)=e^{e^{z}}$ is an entire solution of equation

$$
f^{\prime}(z)=e^{z} f(z+c)^{p}
$$

where $e^{c}=\frac{1}{p}, p \geq 3$ is a positive integer.

Example 3. The function $f(z)=e^{e^{z}}-\frac{1}{e^{e^{z}}}$ is an entire solution of equation

$$
f^{\prime}(z)=2 e^{z}+e^{z} f(z+c)^{2}
$$

where $e^{c}=\frac{1}{2}$.

In what follows, we will consider the existence of solutions of another type DDE, we need the next lemma. We assume $a_{i}(i=0,1, \ldots, p), b_{j}(j=1, \ldots, q)$ are small periodic functions with period $c$ with respect to $f(z)$ in the following.

Lemma 2.4. If $f(z)$ is a transcendental entire function with finite order, then

$$
\begin{aligned}
& T\left(r, f(z)^{n}\left[a_{p} f(z+c)^{p}+\cdots+a_{1} f(z+c)+a_{0}\right]\right) \\
= & (n+p) T(r, f)+S(r, f) .
\end{aligned}
$$

Proof. From Lemma 2.1, we get

$$
\begin{aligned}
& T\left(r, f(z)^{n}\left[a_{p} f(z+c)^{p}+\cdots+a_{1} f(z+c)+a_{0}\right]\right) \\
\leq & n T(r, f)+p T(r, f(z+c))+O(1) \\
= & (n+p) T(r, f)+S(r, f) .
\end{aligned}
$$

On the other hand,

$$
\begin{aligned}
& (n+p) T(r, f) \\
= & (n+p) m(r, f) \\
\leq & m\left(r, f(z)^{n}\left[a_{p} f(z)^{p}+\cdots+a_{1} f(z)+a_{0}\right]\right) \\
= & m\left(r, f(z)^{n}\left[a_{p} f(z+c)^{p}+\cdots+a_{0}\right] \frac{a_{p} f(z)^{p}+\cdots+a_{0}}{a_{p} f(z+c)^{p}+\cdots+a_{0}}\right) \\
\leq & m\left(r, f(z)^{n}\left[a_{p} f(z+c)^{p}+\cdots+a_{0}\right]\right)+m\left(r, \frac{a_{p} f(z)^{p}+\cdots+a_{0}}{a_{p} f(z+c)^{p}+\cdots+a_{0}}\right) \\
\leq & T\left(r, f(z)^{n}\left[a_{p} f(z+c)^{p}+\cdots+a_{0}\right]\right)+S(r, f) .
\end{aligned}
$$

Combining above with (8), then (7) follows. 
From above proof, we see that $f(z)^{n}$ can be replaced by a polynomial of $f(z)$ of degree $n$. However, Lemma 2.4 is not valid for meromorphic functions with finite order, which can be seen by the following two examples.

Example 4. The function $f(z)=\frac{1+e^{z}}{1-e^{z}}, e^{c}=-1$. Then

$$
f(z)[f(z+c)+1]=\frac{2}{1-e^{z}},
$$

hence $T(r, f(z)[f(z+c)+1])=T(r, f)+S(r, f)$. In addition, it is easy to get $a f(z) f(z+c)=a$, thus $T(r, a f(z) f(z+c))=O(1)$, where $a$ is a non-zero constant.

Example 5. The function $f(z)=\tan z, c \neq \frac{m \pi}{2}$, where $m \in \mathbb{N}_{+}$. Then $f(z)[f(z+c) \tan c+1]=f(z+c)-\tan c$, thus

$$
T(r, f(z)[f(z+c) \tan c+1])=T(r, f)+S(r, f) .
$$

Using Lemma 2.4, we have the following result.

Theorem 2.5. There are no transcendental entire solutions $f(z)$ satisfying

$$
\left(a_{n} f(z+c)^{n}+\cdots+a_{1} f(z+c)\right) f^{(k)}(z)=P(z),
$$

where $P(z)$ is a non-zero polynomial, $n \in \mathbb{N}_{+}$.

Proof. Assume that $f$ is a transcendental entire solution, from Lemma 2.4, then

$$
\begin{aligned}
(n+1) T(r, f) & =T\left(r, f(z)\left(a_{n} f(z+c)^{n}+\ldots+a_{1} f(z+c)\right)\right)+S(r, f) \\
& =T\left(r, \frac{f(z)}{f^{(k)}(z)}\right)+S(r, f)+O(\log r) \\
& =T\left(r, \frac{f^{(k)}(z)}{f(z)}\right)+S(r, f)+O(\log r) \\
& \leq T(r, f)+S(r, f)
\end{aligned}
$$

which is impossible.

Lemma 2.6. Let $f(z)$ be a transcendental entire function with finite order and Let $F(z)=f(z)^{n} \frac{a_{p} f(z+c)^{p}+\cdots+a_{1} f(z+c)+a_{0}}{b_{q} f(z+c)^{q}+\cdots+b_{1} f(z+c)+b_{0}}$. If $n+p \geq q$, then

$$
\begin{aligned}
(n+p-q) T(r, f) & \leq T(r, F(z)) \\
& \leq(n+d) T(r, f)+S(r, f) .
\end{aligned}
$$

If $n+p<q$, then

$$
\begin{aligned}
(q-n) T(r, f) & \leq T(r, F(z)) \\
& \leq(n+d) T(r, f)+S(r, f),
\end{aligned}
$$

where $d=\max \{p, q\}$. 
Proof. Using Lemma 2.2, we get

$$
T(r, F) \leq(n+d) T(r, f)+S(r, f) .
$$

On the other hand, if $n+p \geq q$, from Lemma 2.4, we obtain

$$
\begin{aligned}
(n+p+q) T(r, f) & =T\left(r, f(z)^{n}\left[a_{p} f(z+c)^{p}+\cdots+a_{0}\right]\left[b_{q} f(z)^{q}+\cdots+b_{0}\right]\right) \\
& \leq T\left(r, F\left[b_{q} f(z+c)^{q}+\cdots+b_{0}\right]\left[b_{q} f(z)^{q}+\cdots+b_{0}\right]\right) \\
& \leq T(r, F)+2 q T(r, f)+S(r, f) .
\end{aligned}
$$

Thus, $T(r, F) \geq(n+p-q) T(r, f)+S(r, f)$.

If $n+p<q$, let $G=f(z+c)^{n} \frac{a_{p} f(z+c)^{p}+\cdots+a_{1} f(z+c)+a_{0}}{b_{q} f(z+c)^{q}+\cdots+b_{1} f(z+c)+b_{0}}$. Thus, we have

$$
\begin{aligned}
q T(r, f) & =T(r, G)+S(r, f) \\
& \leq T(r, F)+T\left(r, \frac{f(z)^{n}}{f(z+c)^{n}}\right)+O(\log r) \\
& \leq T(r, F)+n T(r, f)+S(r, f) .
\end{aligned}
$$

Remark 2. We hope to get $T(r, F)=(n+d) T(r, f)+S(r, f)$ in Lemma 2.6, if $F$ is an irreducible rational function in $f(z)$ and $f(z+c)$. For example, for a very simple function $F(z)=\frac{f(z)[f(z+c)-1]}{f(z+c)+1}$ is irreducible, can we get $T(r, F)=2 T(r, f)+S(r, f)$ ? However, the following function shows that above question is false. If $f(z)=-\cos ^{2} z$ and $c=\frac{\pi}{2}$, thus $F(z)=1+\sin ^{2} z$. Then we have $T(r, F(z))=T(r, f)=2 T(r, \sin z)+S(r, f)$.

Using the similar method as the proofs of Theorem 2.5 and Lemma 2.6, we have the next result.

Theorem 2.7. If $|n-m|>2$, then there are no transcendental entire solutions with finite order $f(z)$ satisfying

$$
\frac{a_{n} f(z+c)^{n}+\cdots+a_{1} f(z+c)}{b_{m} f(z+c)^{m}+\cdots+b_{1} f(z+c)} f^{\prime}(z)=P(z),
$$

where $P(z)$ is a non-zero polynomial, $n \in \mathbb{N}_{+}$.

$$
\text { 3. Some results on } f^{\prime}(z)=a(z) f(g(z))+b(z) f(z)+c(z)
$$

In this section, we will consider a generally DDE of certain form

$$
f^{\prime}(z)=a(z) f(g(z))+b(z) f(z)+c(z),
$$

which ever be considered by Li and Saleeby [17], also can be seen as the discrete version of scalar linear equation $u^{\prime}(t)+a_{1} u(t)+a_{2} u(t+w)=0$. They obtained the following result.

Theorem B ([17, Theorem 2.4]). Suppose that $f(z)$ is a transcendental meromorphic function and $g(z)$ is an entire function satisfying $(16)$, where $a(z) \not \equiv 0$, $b(z), c(z)$ are polynomials. Then $g(z)$ must be linear. 
From Theorem B, we know that $g(z)$ can be written as $g(z)=a z+b$, where $a$ is a non-zero constant, $b$ is a constant. Here, we will give the following result of the solutions of (16).

Theorem 3.1. Suppose that $f(z)$ is a finite order transcendental entire function of (16) and $g(z)$ is an entire function. If $c(z) \not \equiv 0$, then $\lambda(f)=\sigma(f)$, if $c(z) \equiv 0$ and $\lambda(f)<\sigma(f)$, then $\sigma(f)=1$.

Proof. Assume that $\lambda(f)<\sigma(f)$ provided that $c(z) \not \equiv 0$, then $f(z)$ can be stated as $f(z)=h(z) e^{p(z)}$, where $p(z)$ is a polynomial with $\operatorname{deg}(p(z))=p$ and $\sigma(h)=\lambda(f)<\sigma(f)<+\infty$. Substituting $f(z)$ into (16), we have

$$
\left[h^{\prime}(z)+h(z) p^{\prime}(z)-b(z) h(z)\right] e^{p(z)}-a(z) h(a z+b) e^{p(a z+b)}=c(z) .
$$

Set $f_{1}(z):=\frac{\left[h^{\prime}(z)+h(z) p^{\prime}(z)-b(z) h(z)\right] e^{p(z)}}{c(z)}$ and $f_{2}(z):=\frac{-a(z) h(a z+b) e^{p(a z+b)}}{c(z)}$. Thus, we have $f_{1}(z)+f_{2}(z)=1$. From the second main theorem, we have

$$
T\left(r, f_{1}\right) \leq \bar{N}\left(r, f_{1}\right)+\bar{N}\left(r, \frac{1}{f_{1}}\right)+\bar{N}\left(r, \frac{1}{f_{2}}\right)+S\left(r, f_{1}\right)=S\left(r, f_{1}\right),
$$

which is impossible. Thus, $\lambda(f)=\sigma(f)$.

If $c(z) \equiv 0$, from (17), we have $p(a z+b)-p(z)$ should be a constant, for avoiding a contradiction, $p(z)=z+b$ follows, thus $\sigma(f)=1$.

The differential-difference (or delay-differential) equation $f^{\prime}(x)=f(x-k)$, $k>0$ is well known and extensively studied in real analysis, some results can be found in the book [2]. However, it seems that we have little understanding on meromorphic solutions of complex differential-difference equation

$$
f^{\prime}(z)=f(z+c),
$$

where $c$ is a non-zero complex constant. As we all know that the solution of $f^{\prime}(z)=f(z)$ is of the form $f(z)=A e^{z}$, where $A$ is a constant and the solution of $f(z)=f(z+c)$ can be seen as a periodic function with period $c$. The solutions of (18) exist, for example, $f(z)=e^{z}$ is a solution of (18), where $c=2 k i \pi, k$ is an integer and $f(z)=\sin z$ or $f(z)=\cos z$ are also solutions of (18) for suitable $c$. Noticing that above three functions are of order one, it is reasonable to expect the order of (18) is equal to one. Obviously, the equation (18) has no rational solutions. Using the following lemma, Theorem 3.3 can be obtained immediately.

Lemma $3.2([1])$. Let $f(z)$ be a transcendental meromorphic function of order $\sigma(f)<1, h>0$. Then there exists an $\varepsilon$-set $E$ such that

$$
\frac{f^{\prime}(z+c)}{f(z+c)} \rightarrow 0 \text { and } \frac{f(z+c)}{f(z)} \rightarrow 1 \text { as } z \rightarrow \infty \text { in } \mathbb{C} \backslash E,
$$

uniformly in $c$ for $|c| \leq h$. Further, $E$ may be chosen so that for large $z \notin E$, the function $f$ has no zeros or poles in $|\zeta-z| \leq h$. 
Theorem 3.3. The order of meromorphic solutions of (18) must be satisfy $\sigma(f) \geq 1$.

Before stating another result of the entire solutions of (18), we need the following lemma.

Lemma 3.4. Let $p(z), q(z)$ be non-constant polynomials. If $f(z)$ is a finite order solution of equation

$$
f(z+c)=e^{p(z)}\left(f^{\prime}(z)+q(z) f(z)\right),
$$

then $\sigma(f) \geq \operatorname{deg}(p(z))+1$.

Proof. From (19), we have $\frac{f(z+c)}{f(z)}=e^{p(z)}\left[\frac{f^{\prime}(z)}{f(z)}+q(z)\right]$. Since the lemma of logarithmic derivatives $[12,21]$ and difference analogue of the lemma of logarithmic derivatives [7, Corollary 2.6],

$$
m\left(r, \frac{f^{\prime}(z)}{f(z)}\right)=O(\log r)
$$

and

We have

$$
m\left(r, \frac{f(z+c)}{f(z)}\right)=O\left(r^{\sigma(f)-1+\varepsilon}\right) .
$$

$$
m\left(r, e^{p(z)}\right)=O\left(r^{\operatorname{deg}(p(z))}\right) \leq O\left(r^{\sigma(f)-1+\varepsilon}\right)+O(\log r),
$$

which implies that $\sigma(f) \geq \operatorname{deg}(p(z))+1$.

Theorem 3.5. If $f(z)$ is an entire solution of (18) and $\lambda(f)<\sigma(f)<+\infty$, then $\sigma(f)=1$. Furthermore, one of the following three cases holds:

(i) $f(z)=\left(b_{1} z+b_{0}\right) e^{e z+B}$, where $b_{1}$ is a non-zero constant and $c=\frac{1}{e}$.

(ii) $f(z)=b_{0} e^{A z+B}$, where $c=\frac{\ln |A|+i(\arg A+2 k i \pi)}{A}$ and $A$ is a non-zero constant.

(iii) $f(z)=g(z) e^{A z+B}$, where $g(z)$ is a transcendental entire function and satisfies $g^{\prime}(z)=A[g(z+c)-g(z)]$ and $\sigma(g)<1$, where $A$ is a non-zero constant.

Proof. Since $\lambda(f)<\sigma(f)$, then 0 is a Borel exceptional value of $f(z)$. Thus $f(z)$ must have positive integer order [21, p. 106, Corollary]. Assume that $\sigma(f)=q$, $q$ is a positive integer, then the transcendental entire function $f(z)$ can be written as $f(z)=g(z) e^{Q(z)}$, where $Q(z)$ is a polynomial with $\operatorname{deg}(Q(z))=q$ and $\sigma(g)=\lambda(f)<\sigma(f)<+\infty$. From (18), we have the follows,

$$
\left[g^{\prime}(z)+g(z) Q^{\prime}(z)\right] e^{Q(z)}=g(z+c) e^{Q(z+c)} .
$$

From Lemma 3.4, we have $Q(z+c)-Q(z)=D$, where $D$ is a constant, otherwise $\sigma(g)=\sigma(f)$, a contradiction. Thus, we have $Q(z)=A z+B$, where $A$ is a non-zero constant, $B$ is a constant. Thus $A c=D$ and $\sigma(g)<1$. From (20), we have

$$
g^{\prime}(z)+A g(z)=e^{D} g(z+c)
$$


From Lemma 3.2, we have

$$
\frac{g^{\prime}(z)}{g(z)}+A=e^{D} \frac{g(z+c)}{g(z)}
$$

which implies that $A=e^{D}$. Thus, (21) changes into

$$
g^{\prime}(z)-A[g(z+c)-g(z)]=0 .
$$

If $g(z)$ is a polynomial, then we assume that $g(z)=b_{n} z^{n}+b_{n-1} z^{n-1}+\cdots+$ $b_{1} z+b_{0}$. For avoiding a contradiction with (23), we have $g(z)=b_{1} z+b_{0}$ and $A c=1$, where $b_{1}$ is a non-zero constant or $g(z)=b$ where $b$ is a nonzero constant. If $g(z)=b_{1} z+b_{0}$, then $D=1$ and $A=e$ and $c=\frac{1}{e}$, thus $f(z)=\left(b_{1} z+b_{0}\right) e^{A z+B}$. If $g(z)=b_{0}$, then $e^{A c}=A$, thus $c=\frac{\ln |A|+i(\arg A+2 k i \pi)}{A}$ and $f(z)=b_{0} e^{A z+B}$.

The Borel exceptional polynomial $p(z)$ of $f(z)$ is satisfying

$$
\lambda(f(z)-p(z))=\limsup _{r \rightarrow \infty} \frac{\log ^{+} N\left(r, \frac{1}{f(z)-p(z)}\right)}{\log r}<\sigma(f),
$$

where $\lambda(f(z)-p(z))$ is the exponent of convergence of zeros of $f(z)-p(z)$. If $f(z)-p(z)$ has no zeros, then $p(z)$ is a Picard exceptional polynomial of $f(z)$.

Theorem 3.6. If $f(z)$ is a finite order entire solution of (18), then $f(z)$ has no non-zero Borel exceptional polynomial.

Proof. Suppose that $p(z) \not \equiv 0$ is a Borel exceptional polynomial of $f(z)$, from Hadamard's factorization theorem, we have $f(z)=p(z)+h(z) e^{q(z)}$, where $\sigma(h)=\lambda(f-p)<\sigma(f)<+\infty$. From (18), we have

$$
\left[h^{\prime}(z)+h(z) q^{\prime}(z)\right] e^{q(z)}-h(z+c) e^{q(z+c)}=p(z+c)-p^{\prime}(z)
$$

It is easy to see that $p(z+c)-p^{\prime}(z) \not \equiv 0$. Set $f_{1}(z):=\frac{\left[h^{\prime}(z)+h(z) p^{\prime}(z)\right] e^{q(z)}}{p(z+c)-p^{\prime}(z)}$ and $f_{2}(z):=\frac{-h(z+c) e^{q(z+c)}}{p(z+c)-p^{\prime}(z)}$. Thus, $f_{1}(z)+f_{2}(z)=1$ follows. Using the second main theorem, we have

$$
T\left(r, f_{1}\right) \leq \bar{N}\left(r, f_{1}\right)+\bar{N}\left(r, \frac{1}{f_{1}}\right)+\bar{N}\left(r, \frac{1}{f_{2}}\right)+S\left(r, f_{1}\right)=O\left(r^{\sigma(f)-\varepsilon}\right)+S\left(r, f_{1}\right),
$$

$\varepsilon$ is a positive number, which is impossible. Thus, $p(z) \equiv 0$. Hence $f(z)$ has no non-zero Borel exceptional polynomial.

\section{Differential-difference analogue of Brück conjecture}

Let us recall the Brück conjecture [3] and some results on the conjecture.

Conjecture. If $f$ is a non-constant entire function with hyper-order $\sigma_{2}(f)<$ $+\infty$, where $\sigma_{2}(f)$ is not a positive integer, and if $f$ and $f^{\prime}$ share one finite value $a \mathrm{CM}$, then $f-a=c\left(f^{\prime}-a\right)$ for some constant $c \neq 0$. 
The cases $a=0$ or $a \neq 0$ with the condition $N\left(r, \frac{1}{f^{\prime}}\right)=S(r, f)$ were proved by Brück [3]. The case of $f$ is finite order entire function was proved by Gundersen and Yang [8]. Later on, for functions of infinite order, Chen and Shon [6] proved that the conjecture holds if $\sigma_{2}(f)<\frac{1}{2}$. Recently, some results on difference analogues of Brück conjecture were considered in [14, 16, 18, 19, 20]. Here, we recall the following result with small changes.

Theorem C ([14, Theorem 1]). Let $f$ be an entire function of order $\sigma(f)<2$. If $f(z)$ and $f(z+c)$ share the value a $C M$, then

$$
\frac{f(z+c)-a}{f(z)-a}=\tau
$$

for a non-zero constant $\tau$.

Remark that (25) also can be seen a difference equation, Li, Yang and Yi [18, Theorem 1.1] considered the growth of solutions of the difference equations related on the difference analogue of Brück conjecture which can be stated as follows.

Theorem D. Suppose that $f(z)$ is an entire solution of equation

$$
f(z+c)-a(z)=e^{P(z)}(f(z)-a(z)),
$$

where $P(z)$ is a polynomial and $a(z)$ is an entire function with $\sigma(a)<\sigma(f)<$ $+\infty$. If $\lambda(f(z)-a)<\sigma(f)$, then $\sigma(f)=1+\operatorname{deg}(P)$.

However, the proof of Theorem D in [18] is complicated, we will give a short and different proof of Theorem D. We need the following lemma. Remark that the equation (27) has a small changes with the original version, but the proof can be given words by words in [7, Theorem 9.2].

Lemma 4.1. Let $A_{0}(z), \ldots, A_{n}(z)$ be entire functions such that there exists an integer $l, 0 \leq l \leq n$, such that $\sigma\left(A_{l}\right)>\max _{j \neq l}\left\{\sigma\left(A_{j}\right)\right\}$. If $f(z)$ is a meromorphic solution to

$$
A_{n}(z) f\left(z+c_{n}\right)+\cdots+A_{1}(z) f\left(z+c_{1}\right)+A_{0}(z) f(z)=0,
$$

then $\sigma(f)>\sigma\left(A_{l}\right)+1$.

Proof of Theorem D. Since that $\lambda(f-a)<\sigma(f)$, then from the Hadamard's factorization theorem, we have $f(z)-a(z)=h(z) e^{Q(z)}$, where $\sigma(h)=\lambda(f(z)-$ a) $<\sigma(f)=\operatorname{deg}(Q(z))=q$, where $Q(z)$ is a polynomial. Hence

$$
f(z+c)-a(z)=h(z+c) e^{Q(z+c)}+a(z+c)-a(z) .
$$

From (26), we have

$$
h(z+c) e^{Q(z+c)}+a(z+c)-a(z)=h(z) e^{P(z)+Q(z)} .
$$

If $a(z+c)-a(z) \equiv 0$, we have $h(z+c)=h(z) e^{P(z)+Q(z)-Q(z+c)}$. Remark that $\operatorname{deg}(Q(z)-Q(z+c))=q-1$ and $\operatorname{deg}(P(z)) \leq \operatorname{deg}(Q(z))$, we discuss two cases: 
Case 1: If $q=1$, it implies that $\sigma(f)=1$, then $\operatorname{deg}(P(z))=1$ or $\operatorname{deg}(P(z))=$ 0 . If $\operatorname{deg}(P(z))=1$, from Lemma 4.1, we have $\sigma(h) \geq 2$, which is impossible. If $\operatorname{deg}(P(z))=0$, then $\sigma(f)=1+\operatorname{deg}(P)$ follows.

Case 2: If $q \geq 2$, assume that $\operatorname{deg}(P) \neq q-1$, then $\sigma(h) \geq q$ follows from Lemma 4.1, which is impossible. Thus $\operatorname{deg}(P)=q-1$, implies that $\sigma(f)=1+\operatorname{deg}(P)$.

If $a(z+c)-a(z) \not \equiv 0$, we have

$$
\frac{h(z+c) e^{Q(z+c)}}{a(z)-a(z+c)}-\frac{h(z) e^{P(z)+Q(z)}}{a(z)-a(z+c)}=1,
$$

which can be defined by $f_{1}(z)+f_{2}(z)=1$. From the follows

$$
\frac{f(z+c)-a(z+c)}{a(z)-a(z+c)}=f_{1}(z)
$$

we have $T\left(r, f_{1}\right)=T(r, f(z+c))+S(r, f)=T(r, f)+S(r, f)$ which implies that $\sigma(f)=\sigma\left(f_{1}\right)$. Thus, from the second main theorem, we have

$$
\begin{aligned}
T\left(r, f_{1}\right) \leq & \bar{N}\left(r, f_{1}\right)+\bar{N}\left(r, \frac{1}{f_{1}}\right)+\bar{N}\left(r, \frac{1}{f_{1}-1}\right)+S\left(r, f_{1}\right) \\
\leq & \bar{N}\left(r, \frac{1}{a(z)-a(z+c)}\right)+\bar{N}\left(\frac{1}{h(z+c)}\right) \\
& +\bar{N}\left(r, \frac{1}{h(z)}\right)+S\left(r, f_{1}\right) \\
\leq & 2 T(r, a)+2 T(r, h)+S\left(r, f_{1}\right),
\end{aligned}
$$

which implies that $\sigma\left(f_{1}\right)=q \leq \max \{\sigma(a), \sigma(h)\}$, it is a contradiction with the assumptions. Thus, we have completed the proof of Theorem D.

Remark 3. From the proof above, we see that $a(z)$ in Theorem D should be a periodic function with period $c$.

Finally, we will consider the differential-difference analogue of Brück conjecture. Brück [3] proved that if $f(z)$ is a transcendental entire function and $f(z)$ and $f^{\prime}(z)$ share $0 \mathrm{CM}$, then $f^{\prime}(z)=A f(z)$. Heittokangas etc. [14] obtained that if $f(z)$ is a transcendental meromorphic function with $\rho(f)<2$ and $f(z)$ and $f(z+c)$ share $0 \mathrm{CM}$, then $f(z)=A f(z+c)$. However, taking $f(z)=e^{e^{z}}$ and $e^{c}=1$, thus we have $\frac{f(z+c)}{f^{\prime}(z)}=e^{-z}$. Thus, if $f^{\prime}(z)$ and $f(z+c)$ share $0 \mathrm{CM}$, the equation $f^{\prime}(z)=A f(z+c)$ is also not valid. However, from the next two examples, it seems that for finite order entire functions, we can get a similar result.

Example 6. If $f(z)=e^{z}-1$ and $e^{c}=2$, thus $f^{\prime}(z)$ and $f(z+c)$ share the value $1 \mathrm{CM}$, and $\frac{f^{\prime}(z)-1}{f(z+c)-1}=\frac{1}{2}$. 
Example 7. If $f(z)=e^{z}$ and $e^{c}=2$, thus $f^{\prime}(z)$ and $f(z+c)$ share $0 \mathrm{CM}$, then $\frac{f(z+c)}{f^{\prime}(z)}=2$.

From the above examples, it is interesting to explore the problem if $f^{\prime}(z)$ and $f(z+c)$ share a constant $a \mathrm{CM}$, can we get $\frac{f^{\prime}(z)-a}{f(z+c)-a}=A$, where $A$ is a non-zero constant. For the proof of our result, we need the following lemma, which dues to Yang and Yi, see [21]:

Lemma 4.2 ([21, Theorem 1.56]). Let $f_{1}, f_{2}, f_{3}$ be meromorphic functions such that $f_{1}$ is not a constant. If $f_{1}+f_{2}+f_{3}=1$ and if

$$
\sum_{j=1}^{3} N\left(r, 1 / f_{j}\right)+2 \sum_{j=1}^{3} \bar{N}\left(r, f_{j}\right)<(\lambda+o(1)) T(r),
$$

where $\lambda<1$ and $T(r):=\max _{1 \leq j \leq 3} T\left(r, f_{j}\right)$, then either $f_{2}=1$ or $f_{3}=1$.

Theorem 4.3. Let $f(z)$ be a transcendental entire function with finite order. Suppose that $f(z)$ has a Picard exceptional value $d$ and $f^{\prime}(z)$ and $f(z+c)$ share the constant a $C M$, then $\frac{f^{\prime}(z)-a}{f(z+c)-a}=A$, where $A$ is a non-zero constant. Furthermore, if $a \neq 0$, then $A=\frac{a}{a-d}$.

Proof. Since $f(z)$ has a Picard exceptional value $d$, then we obtain that $f(z)=$ $d+e^{h(z)}$, where $\rho(f)=\operatorname{deg}(h(z))$ and $h(z)$ is a non-constant polynomial. Thus,

$$
f(z+c)=d+e^{h(z+c)}
$$

and

$$
f^{\prime}(z)=h^{\prime}(z) e^{h(z)} .
$$

Since that $f^{\prime}(z)$ and $f(z+c)$ share the constant a CM, then $\frac{f^{\prime}(z)-a}{f(z+c)-a}=e^{p(z)}$, where $p(z)$ is a polynomial and $\operatorname{deg} p(z) \leq \operatorname{deg}(h(z))$. Thus, we have

$$
h^{\prime}(z) e^{h(z)}-(d-a) e^{p(z)}-e^{p(z)+h(z+c)}=a .
$$

Case 1: $a=0$. Then (32) implies that

$$
h^{\prime}(z) e^{h(z)}-d e^{p(z)}-e^{p(z)+h(z+c)}=0 .
$$

If $d=0$, then $h^{\prime}(z) e^{h(z)}=e^{p(z)+h(z+c)}$. Thus, $h^{\prime}(z)$ has no zeros, so $h(z)$ is a polynomial with $\operatorname{deg}(h(z))=1$, then $p(z)$ should be a constant.

If $d \neq 0$, then (33) changes into

$$
\frac{h^{\prime}(z) e^{h(z)-p(z)}}{d}-\frac{e^{h(z+c)}}{d}=1 .
$$

Define $f_{1}(z)=\frac{h^{\prime}(z) e^{h(z)-p(z)}}{d}$ and $f_{2}(z)=\frac{e^{h(z+c)}}{d}$. Hence, $f_{1}(z)-f_{2}(z)=1$. Using the second main theorem, we have

$$
T\left(r, f_{2}\right) \leq \bar{N}\left(r, \frac{1}{f_{2}}\right)+\bar{N}\left(r, \frac{1}{f_{2}+1}\right)+\bar{N}\left(r, f_{2}\right)+S\left(r, f_{2}\right)=S\left(r, f_{2}\right),
$$


which is a contradiction. Thus, we have if $a=0$, then $d=0$ and $p(z)$ reduces to a constant.

Case 2: $a \neq 0$ and $a \neq d$. From Lemma 4.2 and $(32),-e^{p(z)+h(z+c)}=a$ and $h^{\prime}(z) e^{h(z)}-(d-a) e^{p(z)}=0$ or $-(d-a) e^{p(z)}=a$ follows. In the former case, we have $h(z)$ is a polynomial with degree one and $p(z)+h(z+c)$ and $p(z)-h(z)$ are constants simultaneously, thus $p(z)$ should be a constant, then we have $h(z)$ should be a constant, which is a contradiction with $h(z)$ is a polynomial of degree one. In the second case, $e^{p(z)}=\frac{a}{a-d}$, then $p(z)$ is a constant. If $a \neq 0$ and $a=d$, from (32), we get easily a contradiction. Thus, we have completed the proof of Theorem 4.3 .

Using the similar method as the proof of Theorem B, we also can obtain the following result.

Theorem 4.4. Suppose that $f(z)$ is an entire solution of equation

$$
f^{\prime}(z)-a(z)=e^{p(z)}(f(z+c)-a(z)),
$$

where $p(z)$ is a polynomial and $a(z)$ is an entire function with $\sigma(a)<\sigma(f)$. If $\lambda(f(z)-a(z))<\sigma(f)$, then $\sigma(f)=1+\operatorname{deg}(p(z))$.

Proof. Since that $\lambda(f-a)<\sigma(f)$, then from the Hadamard's factorization theorem, we have $f(z)-a(z)=h(z) e^{Q(z)}$, where $\sigma(h)=\lambda(f(z)-a(z))<$ $\sigma(f)=\operatorname{deg}(Q(z))=q$ and $Q(z)$ is a polynomial. Hence

$$
f(z+c)-a(z)=h(z+c) e^{Q(z+c)}+a(z+c)-a(z),
$$

and

$$
f^{\prime}(z)-a(z)=\left[h^{\prime}(z)+h(z) Q^{\prime}(z)\right] e^{Q(z)}+a^{\prime}(z)-a(z) .
$$

From (36), we have

$$
\begin{aligned}
& {\left[h^{\prime}(z)+h(z) Q^{\prime}(z)\right] e^{Q(z)}-h(z+c) e^{P(z)+Q(z+c)}} \\
& -e^{P(z)}(a(z+c)-a(z))=a(z)-a^{\prime}(z)
\end{aligned}
$$

We will discuss two cases in the following.

Case 1. $a(z)-a^{\prime}(z) \equiv 0$. Then (37) changes into

$$
\begin{aligned}
& {\left[h^{\prime}(z)+h(z) Q^{\prime}(z)\right] e^{Q(z)}-h(z+c) e^{P(z)+Q(z+c)}} \\
& -e^{P(z)}(a(z+c)-a(z))=0 .
\end{aligned}
$$

Assume that $a(z+c)-a(z) \not \equiv 0$, from $(38)$, then we have

$$
\frac{\left[h^{\prime}(z)+h(z) Q^{\prime}(z)\right] e^{Q(z)-P(z)}}{a(z+c)-a(z)}-\frac{h(z+c) e^{Q(z+c)}}{a(z+c)-a(z)}=1,
$$

combining above with the second main theorem, we can get a contradiction.

Assume that $a(z+c)-a(z) \equiv 0$, we have

$$
\left[h^{\prime}(z)+h(z) Q^{\prime}(z)\right]=h(z+c) e^{P(z)+Q(z+c)-Q(z)} .
$$


Remark that $\operatorname{deg}(Q(z+c)-Q(z))=q-1$, if $\operatorname{deg}(P(z)) \neq q-1$, then from Lemma 3.4, we have $\sigma(h) \geq q$, which is a contradiction with $\sigma(h)<q$. Thus, $\operatorname{deg}(P(z))=q-1$ follows, it implies that $\sigma(f)=1+\operatorname{deg}(p(z))$.

Case 2. $a(z)-a^{\prime}(z) \not \equiv 0$. It is easy to get $\left[h^{\prime}(z)+h(z) Q^{\prime}(z)\right] e^{Q(z)}$ is not a constant.

Assume that $a(z+c)-a(z) \not \equiv 0$, from (37) and Lemma 4.2, if

$$
\frac{-h(z+c) e^{P(z)+Q(z+c)}}{a(z)-a^{\prime}(z)} \equiv 1,
$$

thus $\left[h^{\prime}(z)+h(z) Q^{\prime}(z)\right] e^{Q(z)} \equiv e^{P(z)}(a(z+c)-a(z))$. Hence, we obtain

$$
e^{Q(z)+Q(z+c)}=\frac{\left[a(z)-a^{\prime}(z)\right][a(z+c)-a(z)]}{-h(z+c)\left[h^{\prime}(z)+h(z) Q^{\prime}(z)\right]} .
$$

Remark that $\operatorname{deg}[Q(z)+Q(z+c)]=\operatorname{deg}(Q(z))$, the above equation implies that $\sigma(f) \leq \max \{\sigma(h), \sigma(a)\}$, it is a contradiction with the condition of Theorem 4.4. If $\frac{-e^{P(z)}(a(z+c)-a(z))}{a(z)-a^{\prime}(z)} \equiv 1$, from (37), we have

$$
\left[h^{\prime}(z)+h(z) Q^{\prime}(z)\right]=h(z+c) e^{P(z)+Q(z+c)-Q(z)} .
$$

The similar discussions in Case 1 , we have $\sigma(f)=1+\operatorname{deg}(p(z))$.

Assume that $a(z+c)-a(z) \equiv 0$, from (37) and the second main theorem for three small functions [12, Theorem 2.5], we can get a contradiction. Thus, the proof of Theorem 4.4 is completed.

Acknowledgements. The authors would like to thank the referee for valuable suggestions to improve the paper.

\section{References}

[1] W. Bergweiler and J. K. Langley, Zeros of difference of meromorphic functions, Math. Proc. Cambridge Philos. Soc. 142 (2007), no. 1, 133-147.

[2] R. Bellman and K. L. Cooke, Differential-Difference Equations, Academic Press, New York, 1963.

[3] R. Brück, On entire functions which share one value $C M$ with their first derivative, Results Math. 30 (1996), no. 1-2, 21-24.

[4] Z. X. Chen, Growth and zeros of meromorphic solution of some linear difference equations, J. Math. Anal. Appl. 373 (2011), no. 1, 235-241.

[5] Z Z Zeros of entire solutions to complex linear difference equations, Acta Math. Sci. Ser. B Engl. Ed. 32 (2012), no. 3, 1141-1148.

[6] Z. X. Chen and K. Shon, On conjecture of R. Brück concerning the entire function sharing one value CM with its derivative, Taiwanese J. Math. 8 (2004), no. 2, 235-244.

[7] Y. M. Chiang and S. J. Feng, On the Nevanlinna characteristic of $f(z+\eta)$ and difference equations in the complex plane, Ramanujan J. 16 (2008), no. 1, 105-129.

[8] G. Gundersen and L. Z. Yang, Entire functions that share one value with one or two of their derivatives, J. Math. Anal. Appl. 223, (1998), no. 1, 88-95.

[9] R. G. Halburd and R. J. Korhonen, Difference analogue of the lemma on the logarithmic derivative with applications to difference equations, J. Math. Anal. Appl. 314 (2006), no. $2,477-487$. 
[10] _ Nevanlinna theory for the difference operator, Ann. Acad. Sci. Fenn. Math. 31 (2006), no. 2, 463-478.

[11] Meromorphic solutions of difference equations, integrability and the discrete Painlevé equations, J. Phys. A 40 (2007), no. 6, 1-38.

[12] W. K. Hayman, Meromorphic Functions, Oxford at the Clarendon Press, 1964.

[13] J. Heittokangas, R. Korhonen, I. Laine, J. Rieppo, and K. Tohge, Complex difference equations of Malmquist type, Comput. Methods Funct. Theory 1 (2001), no. 1, 27-39.

[14] J. Heittokangas, R. Korhonen, I. Laine, J. Rieppo, and J. L. Zhang, Value sharing results for shifts of meromorphic functions, and sufficient conditions for periodicity, J. Math. Anal. Appl. 355 (2009), no. 1, 352-363.

[15] I. Laine, Nevanlinna Theory and Complex Differential Equations, Walter de Gruyter, Berlin-New York, 1993.

[16] S. Li and Z. S. Gao, Entire functions sharing one or two finite values CM with their shifts or difference operators, Arch. Math. (Basel) 97 (2011), no. 5, 475-483.

[17] B. Q. Li and E. G. Saleeby, On solutions of functional differential equations $f^{\prime}(x)=$ $a(x) f(g(x))+b(x) f(x)+c(x)$ in the large, Israel J. Math. 162 (2007), 335-348.

[18] X. M. Li, X. Yang, and H. X. Yi, Entire functions sharing an entire function of smaller order with their shifts, Proc. Japan Acad. Ser. A Math. Sci. 89 (2013), no. 2, 34-39.

[19] X. M. Li, H. X. Yi, and C. Y. Kang, Notes on entire functions sharing an entire function of a smaller order with their difference operators, Arch. Math. (Basel) 99 (2012), 261270 .

[20] K. Liu and L. Z. Yang, Value distribution of the difference operator, Arch. Math. (Basel) 92 (2009), no. 3, 270-278.

[21] C. C. Yang and H. X. Yi, Uniqueness Theory of Meromorphic Functions, Kluwer Academic Publishers, 2003.

KAI LIU

Department of Mathematics

NANCHANG UNIVERSITY

NAnChAng, JiAngxi, 330031, P. R. China

E-mail address: liukai418@126.com

XiAnJing Dong

Department of Mathematics

NANCHANG UNIVERSITY

NANChAnG, JiangXi, 330031, P. R. China

E-mail address: 1713618223@qq.com 